\title{
A!
}

This is an electronic reprint of the original article.

This reprint may differ from the original in pagination and typographic detail.

Sevsek, David; Hinkkanen, Marko; Kukkola, Jarno; Lehtonen, Matti

\section{Dynamic Model of a Virtual Air Gap Variable Reactor}

Published in:

2021 IEEE PES Innovative Smart Grid Technologies Europe (ISGT-Europe)

DOI:

10.1109/ISGTEurope52324.2021.9640077

Published: 01/01/2021

Document Version

Peer reviewed version

Please cite the original version:

Sevsek, D., Hinkkanen, M., Kukkola, J., \& Lehtonen, M. (2021). Dynamic Model of a Virtual Air Gap Variable Reactor. In 2021 IEEE PES Innovative Smart Grid Technologies Europe (ISGT-Europe) IEEE.

https://doi.org/10.1109/ISGTEurope52324.2021.9640077

This material is protected by copyright and other intellectual property rights, and duplication or sale of all or part of any of the repository collections is not permitted, except that material may be duplicated by you for your research use or educational purposes in electronic or print form. You must obtain permission for any other use. Electronic or print copies may not be offered, whether for sale or otherwise to anyone who is not an authorised user. 


\section{Dynamic Model of a Virtual Air Gap Variable Reactor}

\author{
$1^{\text {st }}$ David Sevsek \\ Department of Electrical Engineering and Automation \\ Aalto University \\ Espoo, Finland \\ david.sevsek@aalto.fi \\ $3^{\text {rd }}$ Jarno Kukkola \\ Department of Electrical Engineering and Automation \\ Aalto University \\ Espoo, Finland \\ jarno.kukkola@aalto.fi
}

\author{
$2^{\text {nd }}$ Marko Hinkkanen \\ Department of Electrical Engineering and Automation \\ Aalto University \\ Espoo, Finland \\ marko.hinkkanen@aalto.fi \\ $4^{\text {th }}$ Matti Lehtonen \\ Department of Electrical Engineering and Automation \\ Aalto University \\ Espoo, Finland \\ matti.lehtonen@aalto.fi
}

\begin{abstract}
This paper presents a dynamic model of a virtual air gap variable reactor (VGR). In contrast to traditionally mechanically operated variable reactors, which have been used as arc suppression coils in compensated networks for centuries, VGRs have shown an enhanced response speed. However, the harmonics created by VGRs are considerably higher than those created by mechanically operated reactors. This study's dynamic model considers the magnetic saturation behavior of VGRs, enabling a detailed examination of VGRs. Furthermore, the model can be utilized to develop an effective controller for a VGR. A VGR prototype has been used to validate the dynamic model. It is shown that the dynamic model replicates the actual behavior of a VGR well.
\end{abstract}

Index Terms-Dynamic model, finite element method (FEM), flux-linkage, virtual air gap (VAG), variable reactor.

\section{INTRODUCTION}

Variable reactors have been used in power systems for a long time. Central variable reactor applications include arc suppression coils (ASC) which protect the network in single line-to-earth fault situations. Mechanically controlled arc suppression coils have shown sound arc extinguishing results in the past. In addition, the absence of harmonics while changing the reactor inductance and low costs gave them an essential role in compensated networks. However, although mechanically controlled reactors are valuable tools within compensated networks, their slow response speed is a drawback.

In recent years, there has been an increasing interest in utilizing the virtual air gap (VAG) concept to adjust the inductance of reactors [1]-[3]. The VAG principle relies on auxiliary windings merged into a magnetic core of a reactor. The auxiliary windings are typically excited with DC currents to locally saturate the magnetic core, thereby influencing the core's reluctance. This change in the core's reluctance influences the magnetic flux seen from the primary winding.
An AC voltage supplies the primary winding, and the altered primary flux linkage results in a change in the reactor's inductance. Hence, the magnetic core's reluctance can be modified by controlling the auxiliary winding current. In contrast to mechanically controlled reactors, the utilization of powerelectronically controlled VAGs offers improved response speed and accuracy, potentially enhancing the power system stability.

Several systematic studies of the VAG principle and its application in variable reactors have been undertaken [2], [4]-[6]. Although several researchers investigated the VAG method, only a few attempts have been made to construct analytical models that could help to investigate the operational performance of variable reactors utilizing the VAG method. Such attempts include the dynamic model formulated in [2]. This dynamic state-space model has been validated with measurements of a prototype and has shown satisfactory results. However, the model fails to model the 3rd harmonics. Another analytical modeling approach presented in [7] serves instead as a design tool using reluctance networks. Others [4], [5], [8], [9] utilize the finite element method (FEM) either to analyze magnetic characteristics, the arrangement of VAGs in the magnetic core, or to determine the length of virtual airgaps. Except in [2], none of the previously mentioned studies captured the electrical dynamics of VGRs comprehensively (i.e., no time-domain analysis). However, a detailed dynamic model is essential to investigate both the harmonic content produced by the reactor and its response speed.

Therefore, this paper develops a dynamic analytical model for a virtual air gap variable reactor (VGR) based on basic electromagnetic modeling principles [10], [11]. As in [12], an augmented core-loss model is incorporated into the model to account for losses. Also, the model could be fitted to the prototype VGR, which was developed as part of this study. The dynamic model utilizes data from FEM simulations. Prototype 


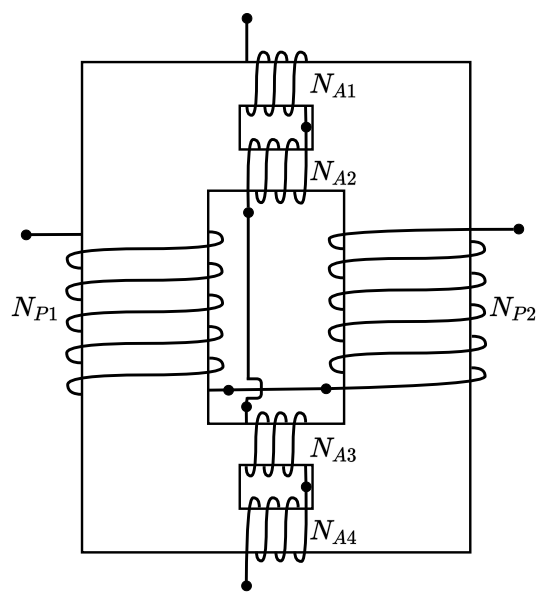

(a)

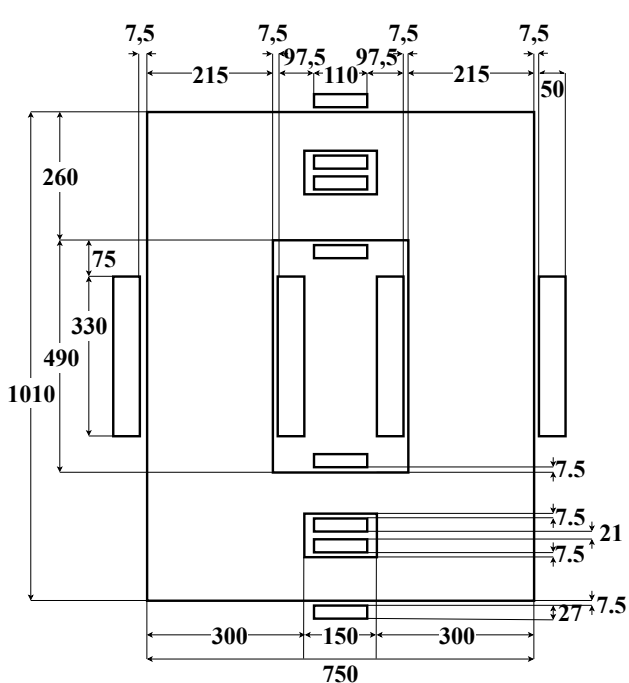

(b)

Fig. 1: VGR prototype: (a) Primary $\left(N_{P 1}=N_{P 2}=346\right.$ turns) and auxiliary $\left(N_{A 1}=N_{A 2}=N_{A 3}=N_{A 4}=200\right.$ turns $)$ coil configuration. (b) Dimensions in millimeters (Core depth $=213.6 \mathrm{~mm}$ ).

tests validate the model's accuracy. The model's intended usages are harmonic analysis, time-domain simulations, and the design of a real-time controller for VAG reactors.

\section{VGR PROTOTYPE}

In [13], an innovative auxiliary winding placement was recommended as indicated in Fig. 1a. In contrast to research in [4], [5], [9], the auxiliary DC control windings are not entirely immersed into the core. Nevertheless, theoretically this solution shall produce the same virtual-air-gap phenomena. As part of this study, a prototype reactor was built following this approach to investigate its electromagnetic behavior. The dimensions of the prototype can be seen in Fig. $1 \mathrm{~b}$.

The advantage of this winding placement is an increased cross-sectional area, meaning that the magnetic flux path increases in the auxiliary winding vicinity. An increased path can be advantageous in high-power applications due to potentially lower flux densities, reducing the reactor's losses.

\section{VGR DYNAMIC MODEL}

\section{A. Saturation Characteristics}

Generally, the induced voltage in a winding together with the voltage drop in the resistance $R$ must balance the applied voltage $u$ as

$$
u=R i+\frac{d \psi}{d t}
$$

where $i$ describes the current through the winding, and $\psi$ is the flux linkage of the winding. Since a VGR consists of primary and auxiliary (i.e., secondary) windings, a dynamic model of a VGR can be defined as

$$
\begin{aligned}
& \frac{d \psi_{1}}{d t}=u_{1}-R_{s, 1} i_{1} \\
& \frac{d \psi_{2}}{d t}=u_{2}-R_{s, 2} i_{2},
\end{aligned}
$$

where $\psi_{1}$ and $\psi_{2}$ describe the primary and secondary flux linkages of the primary and secondary windings, respectively. $R_{s, 1}$ and $R_{s, 2}$ are the series resistances of the primary and secondary circuits, and $u_{1}$ and $u_{2}$ the applied voltages to the respective primary and auxiliary windings. In the case of a VGR, $\psi_{1}$ and $\psi_{2}$ are interconnected through $i_{1}$ and $i_{2}$ as

$$
\begin{aligned}
& \psi_{1}=\psi_{1}\left(i_{1}, i_{2}\right) \\
& \psi_{2}=\psi_{2}\left(i_{1}, i_{2}\right) .
\end{aligned}
$$

The flux linkages $\psi_{1}$ and $\psi_{2}$ can be obtained from FEM simulations, thereby capturing all the system dynamics. The FEM model utilized in this study will be elaborated in Section IV. The dynamic VGR model utilizes the inverted results of the FEM simulation as

$$
\begin{aligned}
& i_{1}=i_{1}\left(\psi_{1}, \psi_{2}\right) \\
& i_{2}=i_{2}\left(\psi_{1}, \psi_{2}\right) .
\end{aligned}
$$

By inverting the FEM results, the dynamic model can directly utilize look-up tables or explicit functions. Since the currents depend on the fluxes as shown in (4), a complete dynamic model can be constructed utilizing (4) and (2) as shown in Fig. 2.

\section{B. Inclusion of Hysteresis and Eddy Current Losses}

Steady-state losses can typically be modeled as

$$
P_{F e}=P_{F t}+P_{H y}=\frac{\omega^{2} \Psi^{2}}{R_{F t}}+\alpha \frac{\omega \Psi^{2}}{R_{F t}},
$$




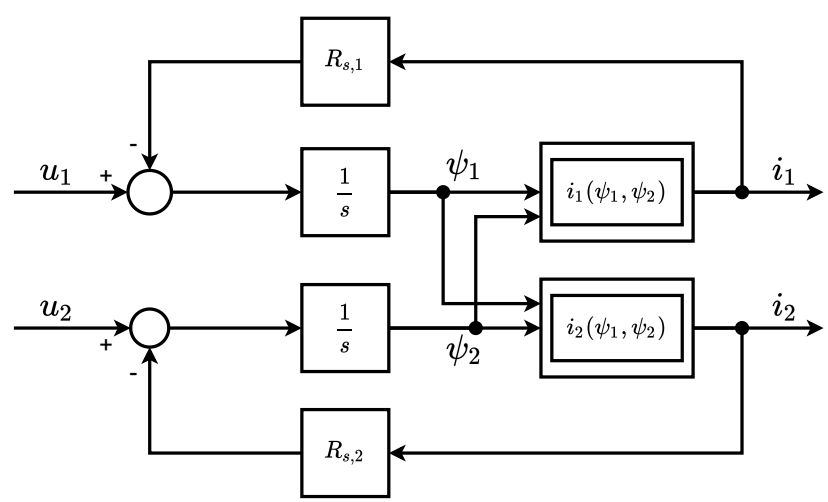

Fig. 2: Schematic representation of the dynamic model of the VGR.

where $P_{F t}$ denotes eddy-current losses, $P_{H y}$ are the hysteresis losses, $\alpha$ determines the ratio between eddy-current and hysteresis losses, and $R_{F t}$ is a constant resistance which relates to the eddy-current losses. Based on (5), in [12], a dynamic loss-model has been developed which enables the inclusion of both loss components. In this model, the instantaneous core-loss current is modeled as

$$
i_{R}=g(u, \psi)=\frac{u}{R_{F t}}+\frac{\alpha|\psi| \operatorname{sgn}(u)}{R_{F t}},
$$

which describes a nonlinear resistance. This dissipation function has been utilized in its reciprocal form where the dissipation is a function of the voltage over the nonlinear resistance, as presented in [12]. The primary voltage drop over the nonlinear resistance can be written as

$$
\begin{aligned}
u_{R, 1} & =h\left(i_{R, 1}, \psi_{1}\right) \\
& =\left\{\begin{array}{cc}
0, & \text { if }\left|i_{R, 1}\right| \leq \frac{\alpha_{1}\left|\psi_{1}\right|}{R_{F t, 1}} \\
R_{F t, 1}\left[i_{R, 1}-\frac{\alpha_{1}\left|\psi_{1}\right| \operatorname{sgn}\left(i_{R, 1}\right)}{R_{F t, 1}}\right], & \text { otherwise. }
\end{array}\right.
\end{aligned}
$$

The secondary side can be modeled in the same way. This form allows modeling the core losses combined with a nonlinear inductor model like the VGR dynamic model. The loss-model can be combined with the series resistances $R_{s}$ of the dynamic VGR model and augmented with a series inductances $L_{s}$, as described in [12].

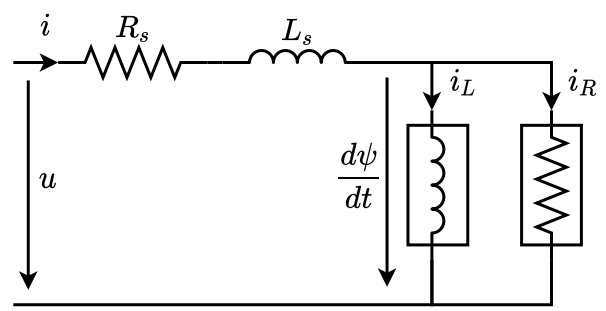

Fig. 3: Augmented equivalent circuit of the nonlinear VGR inductance and the nonlinear core-loss resistance.
TABLE I

VGR Loss Model Parameters

\begin{tabular}{lcl}
\hline \hline \multicolumn{3}{c}{ Primary side } \\
\hline Parameter & Value & Unit \\
\hline$R_{s, 1}$ & 0.405 & $\Omega$ \\
$R_{F t, 1}$ & 9500 & $\Omega$ \\
$L_{s, 1}$ & 0.28 & $\mathrm{H}$ \\
$\alpha_{1}$ & 120 & $\mathrm{rad} / \mathrm{s}$ \\
\hline \multicolumn{3}{c}{ Secondary side } \\
\hline Parameter & Value & Unit \\
\hline$R_{s, 2}$ & 1.157 & $\Omega$ \\
$R_{F t, 2}$ & 1000 & $\Omega$ \\
$L_{s, 2}$ & 0.01 & $\mathrm{H}$ \\
$\alpha_{2}$ & 10 & $\mathrm{rad} / \mathrm{s}$ \\
\hline \hline
\end{tabular}

The resulting equivalent circuit of the VGR model, including the nonlinear resistor for the primary and secondary side, is shown in Fig. 3. The VGR model parameters utilized in this study were empirically determined. Table I displays the chosen model parameters. The model dynamics fitted the actual prototype behavior by selecting those parameters, as shown in section V-B. However, utilizing a cost function to determine the optimal parameters could result in an enhanced model accuracy.

\section{Complete Model Structure}

The dynamic VGR model illustrated in Fig. 2 and the augmented loss-model displayed in Fig. 3 can finally be combined to a complete dynamic model, which includes the entire system dynamics. A block diagram of the complete model structure can be seen in Fig. 4.

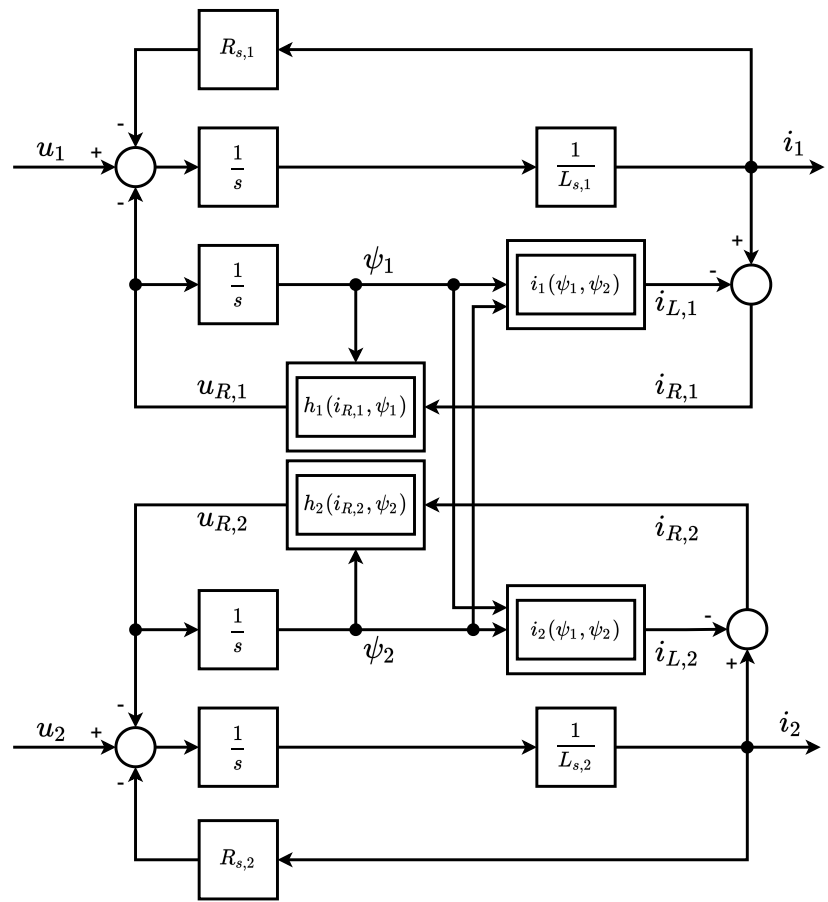

Fig. 4: Block diagram of the complete VGR dynamic model including the augmented core-loss model. 


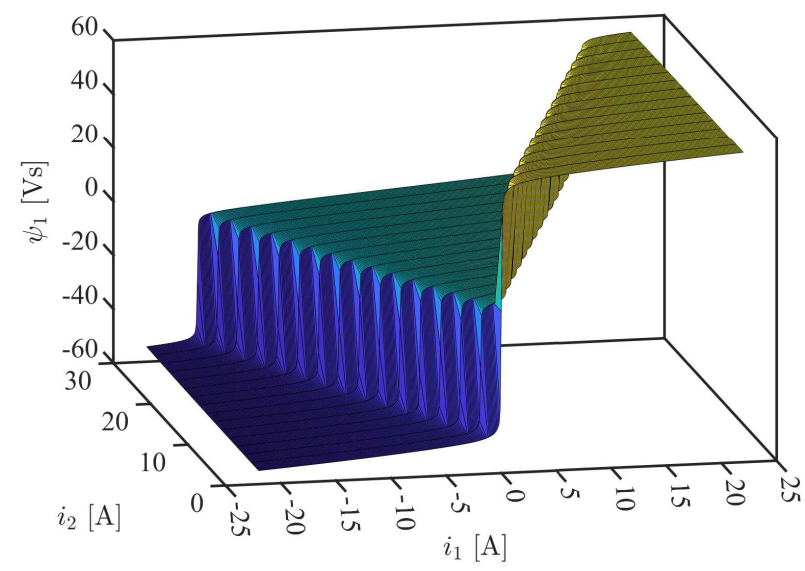

(a)

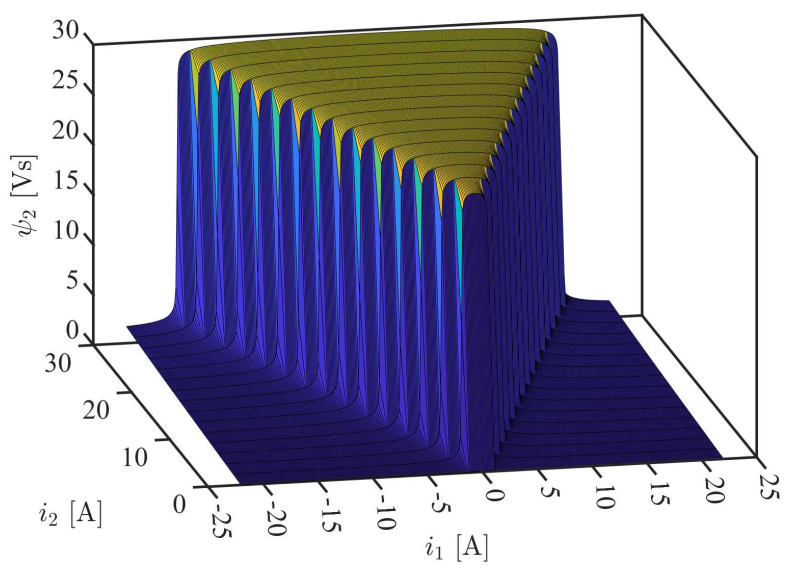

(b)

Fig. 5: Saturation characteristics of the VGR. (a) $\psi_{1}\left(i_{1}, i_{2}\right)$. (b) $\psi_{2}\left(i_{1}, i_{2}\right)$. The saturation characteristics were attained from the FEM simulation model.

\section{FEM MODEL}

The basis of the dynamic VGR model is a large number of magnetostatic FEM simulations. The FEM simulation results capture the magnetostatic behavior of the VGR prototype, hence, providing information about the saturation characteristics of the VGR and eventually about the flux linkages of primary and secondary windings. The FEM simulations have to cover a wide range of primary and secondary current combinations to attain sufficient flux linkage data for the dynamic model as presented in (3). To investigate the VGR prototype and validate the dynamic model, a FEM model was created utilizing the VGR prototype dimensions as indicated in Fig. 1b. The results of the FEM simulations are shown in 5 .

An essential aspect of magnetostatic FEM simulations is the magnetization characteristic of the core. The measured magnetization curve utilized for the FEM model is shown

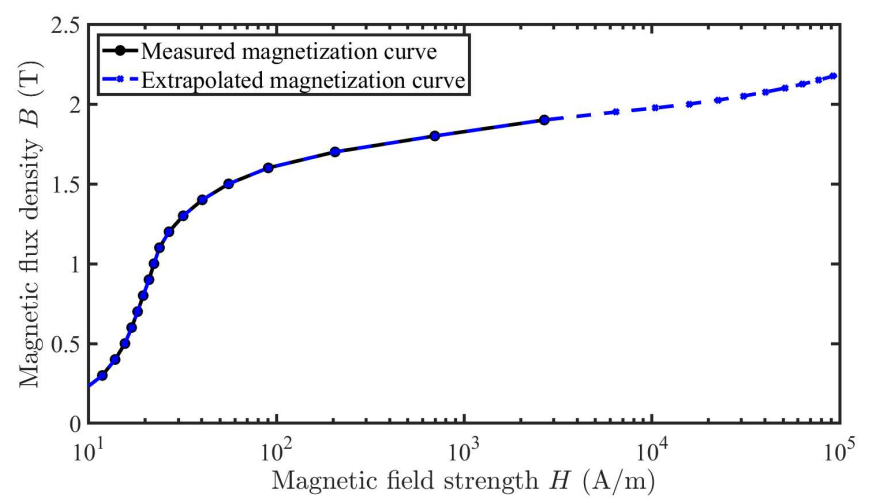

Fig. 6: Magnetization of the core material as a function of the applied field. in Fig. 6. However, the actual measured data does not cover higher magnetic flux densities. Nevertheless, higher flux densities are of particular interest due to the local saturation phenomena caused by the auxiliary winding. Therefore, a quadratic extrapolation technique presented in [14] was applied to attain a reliable data set for the FEM simulations. This technique utilizes common steel characteristics that are available in datasheets of electrical steels. The VGR prototype core was manufactured from ET 150-30 steel. The resulting extrapolated magnetization curve matched perfectly with the lower magnetic flux density regions' measured data and provided enough data for the FEM simulations for higher flux density regions. The extrapolated magnetization curve can also be seen in Fig. 6.

\section{RESUlts}

\section{A. Experimental Setup}

A series of laboratory tests were performed to investigate the performance of the VGR prototype. The laboratory setup was based on an AC power supply (Haefely DTTS 3-2500) transformed to different test voltage levels via a transformer (Haefely-Hipotronics HHT 7100-50L-121156). The primary side of the VGR was supplied with this AC test voltage. The auxiliary winding was supplied with an Agilent N8757A DC power supply. The Agilent power supply was operated in constant current control mode. A LEM Norma 4000 power analyzer and a Tektronix DPO2014 oscilloscope measured both primary and secondary currents and voltages. The currents were measured utilizing Hameg HZ56 current probes. The voltages were determined with Testec differential probes. Some of the measurement results are shown in the subsequent section in which the prototype behavior is compared to the dynamic simulation model results. 


\section{B. Comparison Between Measurements and Simulations}

The dynamic model was validated in a two-step verification process. The first step was a steady-state analysis of simulation and prototype test results. In the second stage, an indepth time-domain analysis of simulation and test results was performed. Both verification steps are elaborated subsequently.

1) Steady-State Analysis: The model accuracy was first evaluated using RMS current and voltage results from both measurement and simulation results. Therefore, the dynamic model was simulated in MATLAB/Simulink at the different primary voltage $u_{1}$ levels (i.e., the primary voltage levels used during the prototype test). In addition, a simple PI controller was utilized to control the secondary side voltage $u_{2}$ so that the secondary RMS currents of the model resembled the measured secondary RMS currents obtained during the tests. Afterward, the primary RMS currents of the model and the tests were compared. Fig. 7 illustrates the difference between the simulated and the actual test results. The primary inductance $L_{1}$ values in Fig. 7b have been calculated as

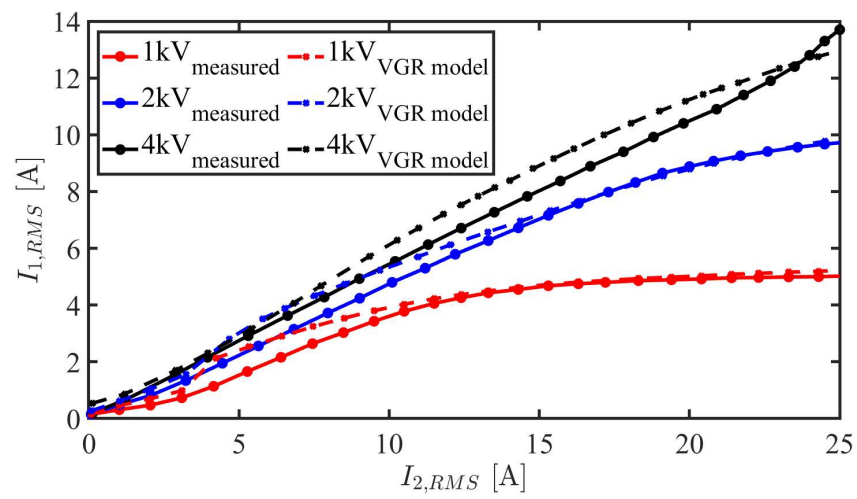

(a)

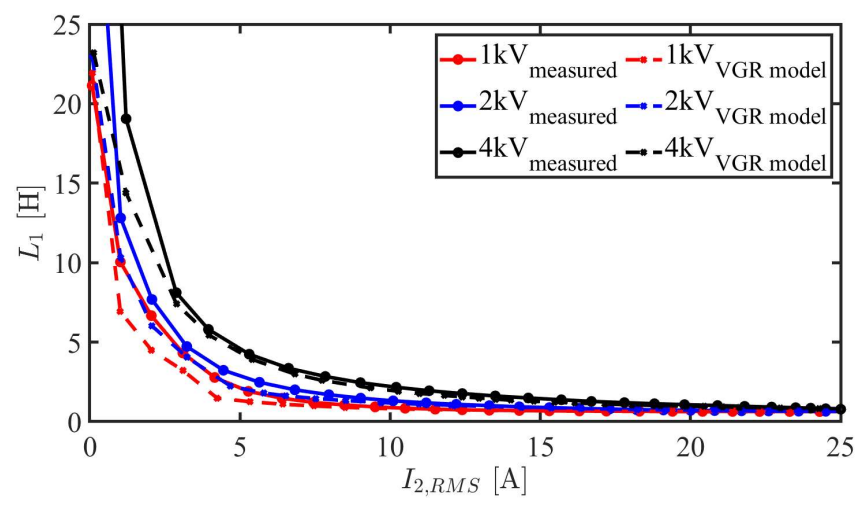

(b)

Fig. 7: Measured (solid line) and simulated (dashed line) results at different primary voltage levels. (a) Primary current as a function of the secondary control current. (b) Primary inductance of the VGR as a function of the secondary control current.

$$
L_{1}=\frac{V_{1, r m s}}{\omega I_{1, r m s}},
$$

where $\omega$ is the fundamental angular frequency of the primary voltage (i.e., $\omega=2 \pi \cdot 50 \mathrm{~Hz}$ ).

Fig. 7 shows that the dynamic model results agree with the actual behavior of the VGR.

2) Time-Domain Analysis: In the second step, the dynamic model was validated, comparing dynamic time-series results. Therefore, the dynamic model was fed with time-series data (i.e., $u_{1}$ and $u_{2}$ ) attained from the oscilloscope during the tests. The simulation results were then compared to the measured data. Fig. 8a illustrates a steady-state situation in which the RMS control current is kept constant at $10 \mathrm{~A}$ and the primary
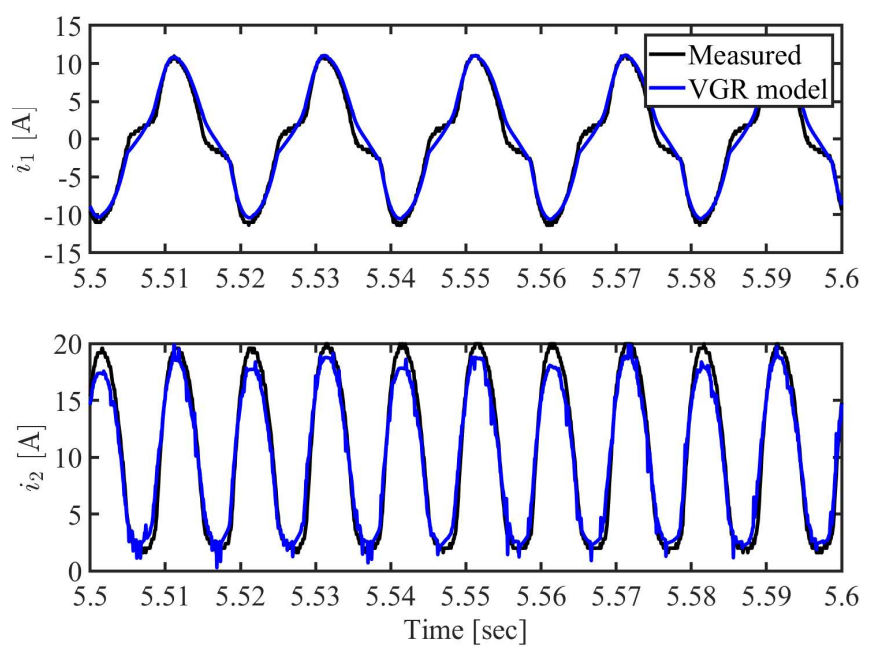

(a)
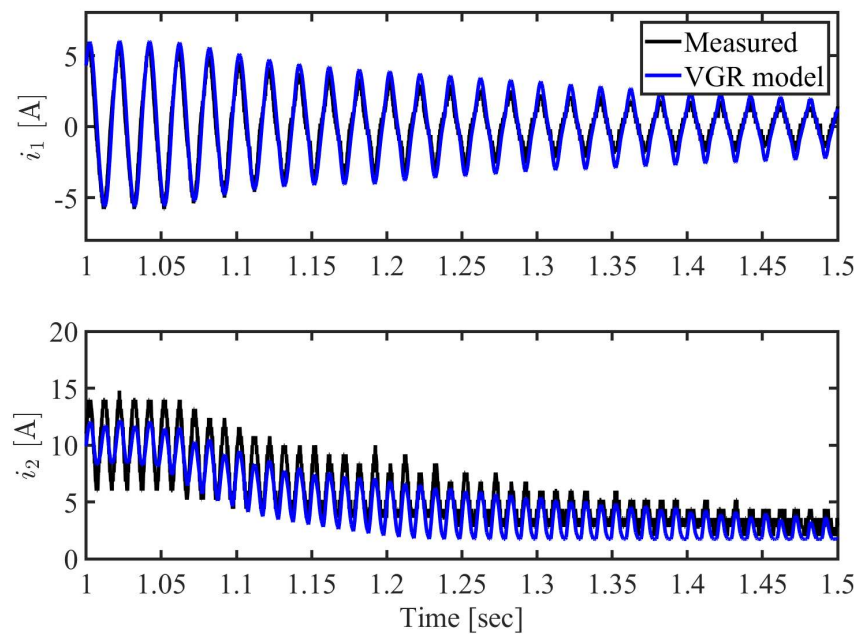

(b)

Fig. 8: Measured and simulated results. (a) Dynamic response at $4 \mathrm{kV}$ and a 10 A RMS control current. (b) Dynamic step response at $1 \mathrm{kV}$ when the RMS control current changes from 10 to $2 \mathrm{~A}$. 
voltage is $4 \mathrm{kV}$. The primary current results of the dynamic model are compatible with the test results. However, the secondary current model results differ marginally from the prototype test results.

Fig. 8b illustrates the behavior of the model and the prototype at a primary voltage of $1 \mathrm{kV}$. In this scenario, the RMS control current changes from 10 to $2 \mathrm{~A}$. It can be seen that the response of the dynamic model resembles the actual prototype. The simulated primary current is consistent with the test results. The model's secondary current magnitudes differ slightly from those measured.

\section{CONCLUSION}

This paper presented a dynamic model of a VGR based on its magnetic saturation characteristics. By incorporating an augmented core-loss model into the basic model, the simulation results were shown to accord with the test results retrieved from a prototype VGR. The deviation of the VGR model simulation results from the test results might have different sources, including measurement inaccuracies in the prototype tests, which result in biased simulation data. Another potential source for the variance may be additional strayflux losses which were perceived during the prototype tests. Furthermore, utilizing a cost function to determine the optimal model parameters could enhance the model accuracy.

Nevertheless, it can be concluded that the dynamic model manages to represent the VGR's system behavior sufficiently. Thus, this model can be utilized in future research work. Such research may include developing appropriate controllers for VGRs and the analysis of VGRs as ASCs in compensated networks.

\section{ACKNOWLEDGMENT}

The authors would like to thank Ensto for the possibility to perform tests on their prototype VGR.

\section{REFERENCES}

[1] J. Penttonen, "Magnetically Controlled Reactor - a Novel Componen in Smart Grid," Ph.D. dissertation, Dept. of Electrical Engineering and Automation, Aalto University, Espoo, Finland, 2018, $56+$ app. 58

[2] D. Dolan, "Modelling and Performance Evaluation of the Virtual Air Gap Variable Reactor," Ph.D. dissertation, University of Toronto, 2009 $102+$ app. 17

[3] V. Molcrette, J.L. Kotny, J.P. Swan, and J.F. Brudny, "Reduction of inrush current in single-phase transformer using virtual air gap technique," IEEE Transactions on Magnetics, vol. 34, no. 4 PART 1, pp. 1192-1194, 1998.

[4] E. Melgoza, J. Avila-Montes, and M. Madrigal, "Analysis of the magnetic characteristics of virtual-gap reactors," in 2013 IEEE International Autumn Meeting on Power, Electronics and Computing, ROPEC 2013

[5] J. Avila-Montes, D. Campos-Gaona, E.M. Vazquez, and J.R. RodriguezRodriguez, "A novel compensation scheme based on a virtual air gap variable reactor for AC voltage control," IEEE Transactions on Industrial Electronics, vol. 61, no. 12, pp. 6547-6555, Dec. 2014.

[6] J. Avila-Montes and E. Melgoza, "Scaling the virtual air-gap principle to high voltage large power applications," in Proc. 20th International Conference on Electrical Machines, pp. 757-762, 2012.

[7] J.F. Brudny, G. Parent, and I. Naceur, "Characterization and Modeling of a Virtual Air Gap by Means of a Reluctance Network," IEEE Transactions on Magnetics, vol. 53, no. 7, Jul. 2017.

[8] S. Magdaleno and C.P. Rojas, "Control of the magnetizing characteristics of a toroidal core using virtual gap," in Proc. IEEE Electronics, Robotics and Automotive Mechanics Conference, pp. 540-545, 2010.
[9] A.A. Abrishami and H. Heydari, "Improved accuracy for finite element modeling in virtual air gap length computation," in 14th International Conference on Environment and Electrical Engineering, pp. 271-274, 2014.

[10] H.H. Woodson and J.R. Melcher, Electromechanical Dynamics: Part I: Discrete Systems, vol. 3, JOHN WILEY \& SONS, 1968.

[11] A.E. Fitzgerald, C. Kingsley, and S.D. Umans, Electric machinery, vol.6, McGraw-Hill, 2003.

[12] M. Ranta, M. Hinkkanen, A. Belahcen, and J. Luomi,"Inclusion of hysteresis and eddy current losses in nonlinear time-domain inductance models," in IECON 2011 - 37th Annual Conference of the IEEE Industrial Electronics Society, 2011, pp. 1897-1902.

[13] J. Penttonen, M. Lehtonen, and S. Muhammad, "Smart grid element: efficient controllable inductance with virtual air gap," IET Generation, Transmission \& Distribution, vol. 12, no. 1, pp. 72-77, 2018.

[14] S.-H. Chai, J.-H. Kim, S.-I. Kim, and J.-P. Hong, "Extrapolating BH Curve Data using Common Electrical Steel Characteristics for High Magnetic Saturation Applications," Journal of Magnetics, vol.20, no.3, pp. 258-264, 2015. 